\title{
Attitudes Emphasized in the Clinical Supervision of Medical Students: An Ethnographic Study in Sweden
}

\author{
Maria Skyvell Nilsson*, ${ }^{*}$, Sandra Pennbrant ${ }^{1}$, Kerstin Nilsson $^{2}$, Bo Samulesson ${ }^{3}$ and Ewa Pilhammar ${ }^{2}$ \\ ${ }^{1}$ The Department of Nursing, Health and Culture, University West, Trollhättan, Sweden \\ ${ }^{2}$ Institute of Health and Care Sciences, The Sahlgrenska Academy, University of Gothenburg, Göteborg, Sweden \\ ${ }^{3}$ Institute of Biomedicine, Department of Clinical Chemistry and Transfusion Medicine, The Sahlgrenska Academy, \\ University of Gothenburg, Göteborg, Sweden
}

\begin{abstract}
Introduction: Medical student's professional attitudes are expected to be developed in medical school, and particularly during clinical education. In this study we focus on supervision in order to describe the attitudes emphasized in the clinical education of fourth-year medical students taking a surgical course.

Methods: An ethnographic approach was applied where observation and interviews were conducted. Nine medical students and twelve supervisors at a teaching hospital in Sweden participated. Field notes were made during observation as well as interviews; these were transcribed and analysed qualitatively.

Result: The analysis resulted in six topic areas describing the attitudes emphasized. The medical students were expected to be: 1) Informed and effective decision makers, 2) Sensitive to patients' needs and expectations, 3) Communicative, 4) Authoritative and patriarchal, 5) Adaptable to organizational demands, and 6) Mindful of nurse's knowledge and requests.

Conclusions: This study reveals that the attitudes emphasised during supervision are: dualistic and complex to learn, developed by a former generation and influence student learning. Students need support in order to handle the state of tension that exists in the attitudes emphasized. Medical students might experience difficulties in adopting some attitudes belonging to a former generation. There is a need for competence development among supervisors concerning how students may experience the attitudes emphasized in supervision.
\end{abstract}

Keywords: Clinical education, clinical learning, hidden curriculum and professional development.

\section{INTRODUCTION}

During clinical education medical students are expected to develop both medical knowledge and attitudes. Medical clinical education involves a gradual assimilation of the knowledge, values, norms and attitudes related to the medical profession. The medical doctors' attitudes emphasized during clinical education are not well described in research literature. Therefore, the aim of this study was to describe attitudes emphasized during supervision.

During medical education, and in particular during clinical education, the student is socialized into the culture of medicine. Professional attitudes are developed and formed along with more experience and knowledge [1], generational belonging [2], and medical specialty [3]. Accordingly, we can assume that medical students will meet and handle attitudes, which have originated in the traditions of medicine and specialty, as well as from supervisors' own personal experience and generational belonging. In medical education, such implicit premises have been acknowledged as the hidden curriculum [4]. Studies have shown that traditions

*Address correspondence to this author at the University West, The Department of Nursing, Health and Culture, Trollhättan, Sweden; Tel: +46520-223878; Fax: +46 520-22 30 99; E-mail: Maria.Skyvell-Nilsson@hv.se and rules have a profound influence on attitudes and behaviour during clinical medical education $[3,5,6]$. Encounters with clinical practice have been shown to play a decisive part in developing attitudes belonging to the profession [7-9] and are considered valuable by students $[7,10,11]$. Nevertheless, students experience clinical practice as complex, and feelings of difficulty in adjusting to the style of learning and teaching [12], difficulty in understanding roles and responsibilities, to adjusting to the culture of patient care in various clinical environments [13], and acquiring professional knowledge skill and attitudes [14], has been described. In the Western world, half of the doctors' workforce will soon consist of women $[15,16]$, although specialist segregation persists, for example in surgery $[17,18]$. The lack of same-sex role models and the existence of gender-based discrimination have been reported as factors that may deter female students from a career in general surgery [18]. Accordingly, in the student's education and socialization into the culture of medicine there is a hidden curriculum, also assumed to include attitudes. Most studies on the subject take the perspective of the student or the supervisor [13, 19-22]. Earlier research does not illuminate the attitudes and in what way they are emphasized during clinical education. To gain a more comprehensive understanding of the clinical learning situation, it is of great importance to study the attitudes empha- 
sized during supervision. The aim of this study is therefore to describe the attitudes emphasized during supervising fourth-year medical students on a surgical course.

\section{METHODS AND MATERIALS}

To be able to study a phenomenon that is often unspoken and sometimes taken for granted, an ethnographic research method seemed appropriate. In ethnographic research [23], the researcher is present in the researched activity. The ethnographic approach is comprehensive, context-bound and reflexive to its nature. This makes it possible to study phenomena in their everyday context, and enables questioning of meaning and interpretations [24].

\section{Setting}

The setting for this study was supervision of fourth-year medical students taking a course in surgery, at a teaching hospital in Sweden (inpatient care). Undergraduate medical students in Sweden study for 11 semesters (5.5 years) before graduating as master of medicine. Clinical practice during this surgical course covered 16 weeks, two of which were spent at a Medical Students' Ward (MSW). At the MSW, according to the official curriculum, supervisors were expected to support students in managing patients, and students were expected to develop intellectual and practical medical skills as well as their professional role. The principal of the medical school and the healthcare directors facilitated access to wards, supervisors and students.

\section{Participants}

Nine medical students (aged 23-37) were purposefully selected by the research group, and asked to participate. The students were selected to represent different age groups, gender and surgical units. Each student was observed on three different occasions where twelve supervisors (aged 36$65)$ participated. In the student group, two men and seven women participated. All students were born and raised in Sweden. The number of female students reflects the actual class of students that year, where about $70 \%$ were women. Eleven of the twelve supervisors were men, which reflect the group of consulting surgeons in the region. Two of the supervisors were raised and educated in other European countries.

\section{Data Collection}

Observations and interviews were used as the data collection methods as opportunities to identify patterns, similarities and differences in the way the supervisors and students acted

I Observations where
working hypotheses
were created and
used for guiding
further observations

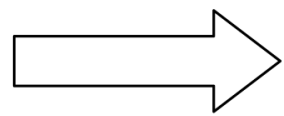

II Interactive analysis process where working hypotheses were confirmed or changed

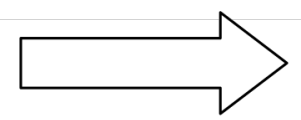

in a context [25]. Data were collected in the year of 2004 and 2005. The observations [27 occasions) were conducted mainly during medical rounds (21 occasions), where students, a supervisor, and a nurse participated within a fourmonth period totalling one hundred hours. A few observations were also carried out at surgical outpatient clinics (3 occasions), in operating rooms (2 occasions) and during clinical lectures (1 occasion) (see Appendix 1). The observations were guided by the aim of the study, and were carried out by two researchers. The two researchers were experienced registered nurses and doctoral students in the field of Health Care Pedagogics. Field notes, including observational notes and analytical memos, were taken during the observations [24]. The researchers conducted observations individually and together. Directly after the observations their individual field notes were compared and interpretations were discussed, to determine agreement, and good agreement was observed. Interviews were conducted in direct connection to the observations, in order to get a broader and deeper understanding of statements or actions, and/or to determine if the actions were representative of an ordinary day. Further documentation was carried out immediately after the observations [24], and the field notes were transcribed after each observation.

\section{Data Analysis}

In accordance with the ethnographic research approach the data collection and the data analysis were conducted simultaneously. An overview of the process of analysis in ethnography is presented in Fig. (1).

The first step ( $\mathrm{I}$ in Fig. 1) of the analysis consisted of making hypotheses [24] concerning the attitudes observed during the observations. These hypotheses were used as preliminary working hypotheses i.e., hypotheses such as, Is this an attitude related to the patient? or Is this an attitude related to organizational demands. These working hypotheses guided further observations and made it possible to explore the attitudes, and detect patterns in its occurrence during further observations. By an iterative analysis process (II in Fig. 1) the working hypotheses were used to make sense of data and data were used to confirm or change hypotheses. The analysis advanced [24], and by continuously comparing with previously collected material, i.e. tested (III in Fig. 1) and the understanding of the phenomena in focus was widened and deepened [26]. While the analysis process advanced, the sentences and paragraphs containing aspects that relate to each other through their content and contextdistinguished and patterns (IV in Fig. 1) could be described as six

\section{IV}

Working hypotheses Patterns of six topic

were tested with areas could be

previouslycollected described material
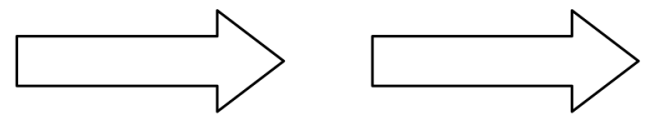

Fig. (1). Illustration of the ethnographic analysis. 
meaningful topic areas. The analysis proceeded and was finalized when all text material was analysed and consistency was ensured. The analysis was discussed in the research group until agreement was reached, and resulted in six topic areas described below.

\section{Ethical Considerations}

Permission to carry out the study was given by the head of each department. Participants were informed of the purpose, method and publication of the study, that participation was voluntary, and that they could withdraw from participation at any time, before informed consent was obtained [27]. According to Swedish law, this type of study does not require approval by an ethics committee. In the observations where patients were present, the patients were informed of the purpose of the study, and that the researchers were bound by professional secrecy in their role as healthcare personnel.

\section{RESULTS}

The results will be presented, together with illustrative field notes. Each field note is marked with an observational code as shown in Appendix I. Attitudes emphasized in supervision were related to expectations for the students to behave and perform. The topic areas of attitudes are described below.

\section{Informed and Effective Decision Makers}

Doctors' responsibilities concerning patients' treatments were made obvious, when the supervisor pointed out the responsibilities of a doctor, for example the ability to make a diagnosis.

Supervisor: Everyone should be able to get a "positive" diagnosis, because that's part of the satisfaction of going to a doctor, isn't it. (O19)

The supervisors also emphasized the doctors' responsibility, to always develop their medical knowledge and understanding of clinical problems as well as to follow scientific research in the specialty. Supervisors also underlined the importance of students having solid theoretical knowledge in order to be able to develop clinical knowledge. Before meeting patients on the round, students were encouraged by their supervisors to act as doctors and take full responsibility when meeting patients. Now you're the doctor, now it's your decision [O4], was a common phrase expressed in several ways during supervision, for example;

The student wonders what approach to take and asks the supervisor. The supervisor counters by saying: Well, you're the one who's supposed to come up with an answer here, and laughed expressing that this was obvious. (O2)

If students indicated lack of knowledge and/or experience when communicating with patients, the supervisor supported them in maintaining their role as knowledgeable doctors. The expectation, of being able to manage clinical patients' problems, was sometimes experienced as too challenging by the medical students. Accordingly, the students were expected to have medical knowledge and be able to make decisions.

Students expressed, in glances and looks, that having to already bear the doctor's responsibility sometimes felt diffi- cult. Similar reactions were observed in situations when supervisors' expectations seemed overwhelming, for example in relation to clinical knowledge.

During the preparatory discussion before the ward round, the supervisor asks the student another question about what she intends to do with the patient. The student seems uncertain and says that she doesn't know what she's going to do. Then the supervisor counters with; "You're the one who's the doctor. You can't turn to anyone else; it's up to you now." "OK, OK," says the student, and seems uncomfortable with the situation. (O3)

\section{Sensitive to Patients' Needs and Expectations}

Supervisors emphasized what they considered to be appropriate attitudes towards the patient, for example: creating trust, creating a relationship, living up to patients' expectations and showing authority.

The supervisor summarizes what has happened and discusses the way the medical students receive and interact with the patients: as a doctor it's important to establish contact with the patient, so that the patient feels secure and can feel confident in me as a doctor, in what I say and do. (O6)

Supervisors also pointed out that it was important to have a holistic view of patients and view "the whole picture", including for example age, gender, state of health, eating habits, psychological components. A doctor should develop a bedside manner [O4]. Consequently, a doctor should try to view things from the perspective and situation of the patient, and with respect to how the patient lives his/her life.

An attitude maintaining that patients should not be seen as one group with the same expectations of doctors' behaviour and that doctors have to consider such differences in patients' expectations was observed. You have to stand up straight and have your coat buttoned up [05]. Such statement reflects a senior supervisor's perception of how patients view a professional doctor.

\section{Communicative}

As a doctor it is essential to be communicative, in order to be able to give and obtain relevant information. The supervisors emphasized both content and performance in communication with patients and that communication skills required deliberate practice to develop. In communication with patients, students were encouraged to be positive and supportive, to call attention to the healthy aspects of the patient, and to view things from the patient's perspective.

At the follow-up, the supervisor emphasizes that the student did well when she said: "You've got rosy cheeks" and that it's important to have a positive attitude and bring out what's healthy in the patient. The student looks happy. (O4)

Sometimes during rounds the supervisors had to take over the conversation, and by doing so demonstrated how communication should be conducted. A dialogue or a discussion with the patient were the supervisor provided relevant 
and professional information and thereby made it clear that the decision was to be taken by the patient him/herself. Such communication was mostly seen with junior supervisors, in discussions concerning sick leave whether it was possible for the patient to go back to work, decisions concerning holiday trips etc. The way in which supervisors seemed to prepare the students for such communication was also observed.

In the preparations before the ward round the supervisor says: Think about whether she can go home. Ask the patient what she does for a living. (O26)

\section{Authoritative and Patriarchal}

The hierarchy in the healthcare organization was visible and preserved when supervisors made a point of indicating the doctor's superior position in relation to other personnel. The field note below illustrates the doctor's power position.

Then the supervisor points out: you're not the nurse's slaves - you're the leaders and the nurse has to follow. (O3)

There were differences in senior supervisors' attitudes to female and male students, and an ambiguity was observed regarding how to treat and relate to the way female students acted and communicated. They also expressed an uncertainty about female students' reactions to their guidance.

During a ward round, a young female student asked a male patient [of the same age] how he was feeling, and the patient countered by asking the student doctor: "How are you feeling?". In this situation, the medical student got embarrassed and uncertain about how to continue the conversation with the patient. The supervisor brings up the situation after the ward round and emphasizes that as a woman she has to be extra careful to put her foot down and steer the conversation. /.../ The supervisor encourages the student to stay in charge when questioning the patient, and not to lose control. (O19)

These senior supervisors considered female students fragile and too sensitive to criticism. Some but not all expressed a feeling of uncertainty and lack of strategies in their communication with female students. In order to handle doctors' tasks, the supervisors tried to give the students advice; for example, that they had to have a more outgoing attitude. Students were encouraged to be creative, full of ideas, and able to take the initiative. You've got to be thick-skinned, help yourself, and not be defeated or get upset on account of the least resistance. [05] The supervisor also pointed out situations that could affect their ability to maintain such an attitude in relation to patients; for example, when encountering patients of the same age and the opposite sex. The students then had to take this advice and adjust to it, in order to be accepted. Sometimes female students expressed that they felt misunderstood and unable to apply such an attitude.

\section{Adaptable to Organizational Demands}

A doctor's work has to be adjusted in accordance with time restrictions, which distinguishes the situation of students from the working conditions of qualified doctors. The supervisors also demonstrated such attitudes during observations when students were not given enough time to finish and complete their task. These situations were experienced as stressful and sometimes condescending among students.

The supervisor rounds off the discussion, steers the conversation on to the next patient and points out that they are already 20 minutes late. "This would have been impossible if the students had had their own practice." (O5)

\section{Mindful of Nurses' Knowledge and Requests}

Nurses were involved in the medical students' clinical practice in several ways, e.g. by taking care of the students on the ward and by showing them around. The students could turn to the nurses if they had practical problems and they supplied the students with information concerning the patients' present physical and social situation, and made it possible for them to make decisions. This expectation concerning the role of nurses was also expressed by the supervisors.

The supervisor emphasizes that you get good information from the nurse, and it's important that you use that information and follow it up with questions until you've got a clear picture.

(O7)

The boundary between the two professions in healthcare was made clear when the nurse showed what she expected of the medical student, for example by directing questions to the students concerning medical problems.

The supervisors also conveyed the limitations of nurses' knowledge and judgments in relation to their own professional judgments. They admitted that nurses' knowledge was important, but not sufficient for a doctor to base a judgment on. This attitude was for example demonstrated when the supervisor showed how to handle nurses' requests.

Student: The nurse wants us to prescribe an analgesic for the patient.

Supervisor: I see, but we can't agree to that. Just think if we alleviate the pain so much that it's difficult for the poor person [another doctor] who has to take care of the patient later, because then he can't make a "correct" assessment. (O5)

\section{DISCUSSION}

The aim of this study was to describe attitudes emphasized in clinical supervision of fourth-year medical students on a surgical course. This study reveals that the attitudes emphasised during supervision are: dualistic and complex to learn, developed by a former generation and influence student learning. These conclusions will be discussed below.

\section{Dualistic and Complex to Learn}

The attitudes emphasized during supervision, stand out as dualistic and complex. The maintenance of a hierarchic order towards students during supervision has previously been described [21, 22]. This study's findings also show that students themselves are expected to adopt such attitudes in order to handle their professional role. The attitudes empha- 
sized could be described as a number of divergent attitudes creating a state of tension that medical students were expected to handle. Firstly, integrating a distant approach (being knowledgeable and able to make decisions) and at the same time being expected to be sensitive to the needs and perspective of the patients. We suggest that both these aspects of a doctor's work are crucial in order to facilitate for good patient care. Therefore it is important that supervisors allow for both aspects to be developed during clinical education and support students in such process. Secondly, an ambiguous attitude to relations and cooperation with nurses was presented. Students were encouraged to make use of nurses' knowledge and competence and at the same time act as superiors and be critical towards their statements and requests. In earlier studies, cooperation between nurses and doctors has been described as complicated [28], and educational program to increase cooperation between nurses and medical students have been emphasized in order to improve the quality of care provided by the doctors and nurses of tomorrow [29]. This study could contribute to such a program by facilitating a better understanding of both the opportunities and difficulties in such cooperation. Understanding of each other's competences and professions are a necessity to meet the requirement of effective communication and team-working healthcare [30].

Students need support and guidance to handle the state of tension that exists in the attitudes, e.g., balancing distance and intimacy; acting knowledgeably while being open to learning; and cooperating with other personnel. The supervisor did not always seem aware of the attitudes emphasized or how they should be taught or learned by the student. Howe states that a core learning principal in professional development is to facilitate reflection [31]. A reflective approach to support the students to reflect over experienced situation in relation to the patient and to the cooperation with the nurses was not found in this study result.

\section{Formed by a Former Generation}

The findings reveal differences between senior and junior supervisors' attitudes in encounters with female students and patients. It has also earlier been found that issues of gender have an obvious influence on female students during their medical education. Babaria and colleagues found that female medical students' interaction with their supervisors were influenced by gendered expectations [32]. Some senior doctors in this study, hold a more authoritative attitude which at times seemed to belong to a former generation [2], i.e., a more authoritarian attitude. Junior supervisors on the other hand demonstrated approaches belonging to a younger generation, for example a more democratic way of discussion medical problems with patients and students. A particularly problematic situation related to generational belonging, was observed between female students and senior supervisors when the supervisor encouraged female students to adopt a more authoritative and patriarchal attitude. Davies has described such attitudes as masculine. She states that masculine qualities in medicine are historically constructed and can be described in terms of: physical and mental strength, competitiveness, individualism and dominance [28]. This result is also in accordance with other findings; describing how female students perceived surgeon's behaviour as paternalistic [12]. The situation of female students could consequently be seen as more complicated, in that they challenge the traditional hierarchy in surgery, where the surgeon is usually a male person with a masculine way of expressing himself [28]. These findings indicate that there is a risk that female students may feel less respected among supervisors and nurses due to the patriarchal tradition, and may choose not to continue with their surgical specialty. Johansson and Hamberg suggest that the increasing segregation in specialties must actively be counteracted, by employing role models of both genders and exploring gender awareness among teachers and students [15]. The socialization process in interaction with supervisors could be experienced as more complicated (complex) by medical students that challenge the traditional role, attitudes and hierarchy order in surgery. We therefore predict that medical students will experience difficulties in adjusting to some professional attitudes belonging to a former generation, or attitudes that exclude persons in relation to gender, religion or culture.

\section{Influencing Students Learning}

The findings show how attitudes emphasized influenced students learning situation. In symbolic interactionism, culture is seen as a shared system of meanings learned and maintained in the context of people interacting [23]. Particularly important and influential people in this process are persons who are given the role as specific others [23]. The supervisors could be given the role of significant other. In interaction with significant others the student takes the attitudes of the other by imagining how they think, feel and define their situation. Using this method, the student is able to conduct (interpret and understand) the supervisor's professional attitudes and behaviour [33]. Through the supervisors' confirmations, or lack of, and adjustments, the professional attitudes and culture is handed over to the student. The result could be interpreted as a socialization process where the student is socialized into the culture of surgical health-care and its traditions by taking the perspective of specific other (the supervisor) [23]. In this study the students were entirely dependent on support from their supervisors, and by responding to their confirmation, or absence of confirmation, and making adjustments, the attitudes were made visible. In interaction with supervisors, students also had to handle more personal expectations concerning the attitudes to be applied. There is a risk that students feel forced into the role of the supervisors, or that they feel cast in the same mould as the supervisor, and not given the opportunity to develop their personal professional approach.

Students were expected to learn about clinical work and at the same time adjust to the attitudes stressed. By adopting the attitude of being an informed and effective decision maker, students had to leave their role as a student and focus on acting as a doctor. The findings of this study show that too much focus on the doctor's role sometimes seemed overly demanding for a fourth-year medical student. This result is in accordance with other studies, describing students feelings of stress related to expectations of being seen as confident and competent in interacting with staff, colleagues and patients during clinical practice [14]. Consequently, we imply that if the expectations on students to act independently are too demanding, with respect to their knowledge and skill, this may in turn limit their learning possibilities. 
Organizational demands were sometimes demonstrated by the supervisors who intervened in the student's performance. Such intervening was sometime made in an insensitive way. The students were in a learning situation, meeting patients or/and personnel, with limited experience and knowledge to handle the situation. When the supervisor intervened in this way the students learning process was interrupted and we observed feelings of being down graded or failure in performance by the students. The situation shows the impotence of focusing students understanding in order to support learning [34] and to create a supportive environment. Several studies have previously stressed the importance of supervisors' approaches in order to support learning [35, 36].

The findings are in accordance with other studies showing that supervisors rarely discuss or analyse problems concerning student learning in clinical teaching [37-39]. We suggest that it is essential to highlight clinical supervisors' awareness of their own attitudes and that the competence and attitudes that are essential for being a good supervisor need to be further discussed at medical schools.

\section{Study Strengths and Limitations}

The purpose of qualitative studies is to gain deeper understanding and knowledge concerning a specific phenomenon in focus [40]. By using a ethnographic approach, data collection and analysis were performed simultaneously, making it possible to enter the field with open issues and continuously deepen the understanding of the attitudes emphasized [24]. Selecting situations with specific rich context, i.e. situations dominated by interaction between supervisor and students, made it possible to determine if the analytical hypotheses have been "saturated"in a relatively short period of time [41].

To ensure validity, triangulation is recommended in ethnography [42]. In the present study, this was managed by comparing the observations of the two researchers. Furthermore, the different experiences of the two researchers and the possibility to compare interpretation from the same observations must be seen to strengthen of both validity and reliability in this study. As a researcher you have to consider, that what participants feel to be appropriate to reveal in some context and circumstances may be inappropriate under other conditions. Therefore, the fact that data collection was conducted at different wards and hospitals strengthens the validity of the study [42]. By using nine different students as "gate keepers" i.e. (participants who give entrance to observational situations) [24], we could observe several supervisors in different surgical units and constellations. Consequently, the purpose to represent different age groups, gender and surgical units was also obtained.

As this study is based on observations from only one medical school in Sweden, the extent to which the findings can be generalized to other medical schools remains to be established. In order to secure the quality of our account, we have presented examples of contextual details that will help readers to assess the degree to which our findings are transferable to other settings. The observations in this study were mainly conducted in surgical wards with particular focus on education. Our results are based on observations and interviews during a limited period of supervision at a surgical ward, and consequently we cannot tell how the identified attitudes affect the students' future role as qualified doctors.

\section{CONCLUSION}

The attitudes emphasised during supervision are: dualistic and complex to learn, developed by a former generation and influence student learning. Students need guidance to be able to handle and understand the state of tension involved in the attitudes emphasized (e.g. balancing distance and intimacy; acting knowledgeably while being open to learning; being dependent and at the same time critical concerning nurses' knowledge and requests). Medical students might experience difficulties in adopting some attitudes belonging to a former generation, or could feel rejected due to the generation and/or the gender to which they belong. Poorly adjusted expectations concerning student'spossibility to adopt the attitudes emphasised, may be experienced as too demanding and limit the student's opportunities to learn.

\section{ACKNOWLEDGEMENTS}

The authors would like to thank all the participants for giving their time to take part in this study.The study was partly funded by the VästraGötaland Region in Sweden.

\section{CONFLICT OF INTEREST}

None declared.

Appendix I. Overview of Observations, Observer Participation and Settings

\begin{tabular}{|c|c|c|c|}
\hline Observation & Supervisor & Student & Setting \\
\hline \hline FO1 & A & I & Surgical outpatient clinic, II* \\
\hline O2** & H & I & Surgical ward (MSW) I* \\
\hline O3 & H & I & Surgical ward (MSW), I \\
\hline O4 & C & II & Surgical ward (MSW), II \\
\hline O5 & C & II & Surgical ward (MSW), II \\
\hline O6 & C & II & Surgical ward (MSW), II \\
\hline O7 & D & III & Surgical ward (MSW), III* \\
\hline O8 & L & III & Surgical ward (MSW), III \\
\hline O9 & K & III & Surgical ward (MSW), III \\
\hline O10 & D & IV & Surgical ward (MSW), III \\
\hline O11 & D & IV & Surgical ward (MSW), III \\
\hline O12 & E & IV & Surgical ward (MSW), III \\
\hline O13** & E & V & Surgical ward (MSW), II \\
\hline O14** & E & V & Surgical ward (MSW), II \\
\hline O15** & E & V & Surgical ward (MSW), II \\
\hline O16 & F & VI & Surgical ward (MSW), I \\
\hline O17 & F & VI & Surgical ward (MSW), I \\
\hline O18 & G & VI & OR, II \\
\hline O19** & H & VII & Surgical ward (MSW), I \\
\hline O20 & I & VII & Surgical ward (MSW), I \\
\hline O21 & I & VII & Surgical ward (MSW), I \\
\hline O22 & B & VIII & Surgical ward (MSW), II \\
\hline O23 & B & VIII & OR, II \\
\hline O24 & J & VIII & Practical lecture, II \\
\hline O24** & E & IX & Surgical ward (MSW), II \\
\hline O26** & E & IX & Surgical ward (MSW), II \\
\hline O27** & E & IX & Surgical ward (MSW), II \\
\hline
\end{tabular}

*Hospital unit, MSW (Medical Students' Ward)

**Participation of two observers in this observation. 


\section{REFERENCES}

[1] Skyvell Nilsson M, Knutsson A, Samuelsson B, Lönnroth P, Pilhammar Andersson E. Läkares erfarenhetsbaserade kunskap. Delrapport 1, Erfarenhetens professionella uttryck. (Eng title: Expression of professional experience). Göteborg: Institutionen för vårdvetenskap och hälsa, Sahlgrenska akademin vid Göteborgs universitet; 2006.

[2] Smith LG. Medical professionalism and the generation gap. Am J Med 2005; 118: 439-42.

[3] Atkinson P. The clinical experience: the construction and reconstruction of medical reality. $2^{\text {nd }}$ ed. Aldershot: Ashgate 1997.

[4] Hafferty FW. Beyond curriculum reform: confronting medicine's hidden curriculum. Acad Med 1998; 73: 403-7.

[5] Becker HS. Boys in white: student culture in medical school. New Brunswick: Transaction 1977.

[6] Merton RK, Reader GG, Kendall PL. The student-physician: introductory studies in the sociology of medical education. Cambridge: Harvard Univ Press; 1957.

[7] Parsell G, Bligh J. Recent perspectives on clinical teaching. Med Educ 2001; 35: 409-14.

[8] Weissmann PF, Branch WT, Gracey CF, Haidet P, Frankel RM. Role modeling humanistic behavior: learning bedside manner from the experts. Acad Med 2006; 81: 661-7.

[9] Stephenson AE, Adshead LE, Higgs RH. The teaching of professional attitudes within UK medical schools: Reported difficulties and good practice. Med Edu 2006; 40: 1072-80.

[10] Dyrbye LN, Harris I, Rohren CH. Early clinical experiences from students' perspectives: a qualitative study of narratives. Acad Med 2007; 82: 979-88.

[11] von Below B, Hellquist G, Rodjer S, Gunnarsson R, Bjorkelund C, Wahlqvist M. Medical students' and facilitators' experiences of an Early Professional Contact course: active and motivated students, strained facilitators. BMC Med Edu 2008; 8: 56.

[12] Seabrook MA. Clinical students' initial reports of the educational climate in a single medical school. Med Edu 2004; 38: 659-69.

[13] O'Brien B, Cooke M, Irby DM. Perceptions and attributions of third-year student struggles in clerkships: do students and clerkship directors agree? Acad Med 2007; 82: 970-8.

[14] Radcliffe C, Lester H. Perceived stress during undergraduate medical training: a qualitative study. Med Edu 2003; 37: 32-8.

[15] Johansson EE, Hamberg K. From calling to a scheduled vocation: Swedish male and female students' reflections on being a doctor. Med Teacher 2007; 29: e1-8.

[16] Kilminster S, Downes J, Gough B, Murdoch-Eaton D, Roberts T. Women in medicine-is there a problem? A literature review of the changing gender composition, structures and occupational cultures in medicine. Med Edu 2007; 41: 39-49.

[17] Dodson TF, Webb AL. Why do residents leave general surgery? The hidden problem in today's programs. Curr Surg 2005; 62: 12831.

[18] Park J, Minor S, Taylor RA, Vikis E, Poenaru D. Why are women deterred from general surgery training? Am J Surg 2005; 190: 1416.

[19] Goldie J, Dowie A, Cotton P, Morrison J. Teaching professionalism in the early years of a medical curriculum: a qualitative study. Med Educ 2007; 41: 610-7.

[20] Ratanawongsa N, Teherani A, Hauer KE. Third-year medical students' experiences with dying patients during the internal medicine clerkship: a qualitative study of the informal curriculum. Acad Med 2005; 80: 641-7.
[21] Lempp H, Seale C. The hidden curriculum in undergraduate medical education: qualitative study of medical students' perceptions of teaching. BMJ 2004; 329: 770-3.

[22] Murakami M, Kawabata H, Maezawa M. The perception of the hidden curriculum on medical education: an exploratory study. Asia Pac Fam Med 2009; 8: 9

[23] Blumer H. Symbolic interactionism: perspective and method. Berkeley: University of California Press 1969.

[24] Hammersley M, Atkinson P. Ethnography: principles in practice. $3^{\text {rd }}$ ed. Milton Park, Abingdon, Oxon; New York: Routledge; 2007.

[25] Pilhammar Andersson E. Etnografi i det vårdpedagogiska fältet: en jakt efter ledtrådar(Eng title: Ethnography in health care pedagogics: A pursuit for clues). Lund: Studentlitteratur 1996.

[26] Glaser BG, Strauss AL. The discovery of grounded theory : strategies for qualitative research. Chicago: Aldine 1967.

[27] MFR. World Medical Association Declaration of Helsinki. Riktlinjer för etisk värdering av medicinsk humanforskning: forskningsetisk policy och organisation i Sverige. (Eng title: The Swedish Research Council's guidelines for the ethical evaluation of medical research on humans). Stockholm: Medicinska Forsknings Rådet; 2003.

[28] Davies K. The body and doing gender: the relations between doctors and nurses in hospital work. Sociol Health Illn 2003; 25: 720-42.

[29] Morison S, Jenkins J. Sustained effects of interprofessional shared learning on student attitudes to communication and team working depend on shared learning opportunities on clinical placement as well as in the classroom. Med Teacher 2007; 29: 464-70.

[30] Nilsson K, Hertting A, Petterson IL, Theorell T. Pride and confidence at work: potential predictors of occupational health in a hospital setting. BMC Public Health 2005; 5: 92.

[31] Howe A. Professional development in undergraduate medical curricula-the key to the door of a new culture? Med Edu 2002; 36: 353-9.

[32] Babaria P, Abedin S, Nunez-Smith M. The effect of gender on the clinical clerkship experiences of female medical students: results from a qualitative study. Acad Med 2009; 84: 859-66.

[33] Allan K. Contemporary social and sociological theory : visualizing social worlds. Thousand Oaks, California: Pine Forge Press 2006.

[34] Ramsden P. Learning to teach in higher education. London: Routledge Falmer; 2003.

[35] Sutkin G, Wagner E, Harris I, Schiffer R. What makes a good clinical teacher in medicine? A review of the literature. Acad Med 2008; 83: 452-66.

[36] Dornan T, Boshuizen H, King N, Scherpbier A. Experience-based learning: a model linking the processes and outcomes of medical students' workplace learning. Med Educ 2007; 41: 84-91.

[37] Seabrook MA. Medical teachers' concerns about the clinical teaching context. Med Educ 2003; 37: 213-22

[38] Kilminster SM, Jolly BC. Effective supervision in clinical practice settings: a literature review. Med Educ 2000; 34: 827-40.

[39] Martens MJ, Duvivier RJ, van Dalen J, Verwijnen GM, Scherpbier AJ, van der Vleuten CP. Student views on the effective teaching of physical examination skills: a qualitative study. Med Educ 2009; 43: 184-91.

[40] Leininger M. Evaluation, criteria and critique of qualitative research studies. In: Morse JM, Ed. Critical issues in qualitative research methods. Thousand Oaks, California: Sage 1994; pp. 95115 .

[41] Jeffrey B, Troman G. Time for ethnography. British Educ Res J 2004; 30: 535-48.

[42] Kimchi J, Polivka B, Stevenson JS. Triangulation: operational definitions. Nurs Res 1991; 40: 364-6.

This is an open access article licensed under the terms of the Creative Commons Attribution Non-Commercial License (http://creativecommons.org/licenses/by-nc/3.0/) which permits unrestricted, non-commercial use, distribution and reproduction in any medium, provided the work is properly cited. 